\title{
How fundamental is the character of thermal uncertainty relations?
}

\author{
F. Pennini* ${ }^{*}$ A. Plastino ${ }^{\dagger}$, and A. R. Plastino ${ }^{\ddagger}$ \\ National University La Plata (UNLP) \& Argentine National Research Council (CONICET) \\ C.C. 727, 1900 La Plata, Argentina
}

\begin{abstract}
We show that thermodynamic uncertainties do not preserve their form if the underlying probability distribution is transformed into an escort one. Heisenberg's relations, on the other hand, are not affected by such transformation. We conclude therefore that the former uncertainty cannot be as fundamental as the quantum one.

KEYWORDS: Fisher information, power-law distributions, escort probabilities, thermal uncertainty.
\end{abstract}

02.50.-r, 89.70.+c, 02.50.Wp, 05.30.Ch

\footnotetext{
*e-mail: pennini@venus.fisica.unlp.edu.ar

${ }^{\dagger}$ Corresponding Author, e-mail: plastino@venus.fisica.unlp.edu.ar

‡e-mail: plastino@sinectis.com.ar
} 


\section{INTRODUCTION}

Thermodynamics "uncertainty" relations have been the subject of much interesting work over the years (see, for instance, [1-3]). An excellent, recent review is that of Uffink \& van Lith [4]. We will be interested here in these uncertainty relations insofar as they are derived by recourse to statistical inference [5], with emphasis upon Mandelbrot's results [2].

Heisenberg's uncertainty relations and Bohr's complementarity principle constitute two pillars of 20-th century science. These two prominent authors have suggested that there is a classical analogue of the complementarity principle, specifically between temperature and energy [6]. Although such ideas have not received general acceptation, several renowned authors have defended them, as exemplified by, among others, Refs. [1-3]. These claims remain still controversial (see $[4,7,8])$.

We wish in this Communication to add a footnote to the controversy by focusing attention upon particular aspects of the thermal uncertainty derivation of Mandelbrot's [2]. This derivation contains as an essential ingredient the information measure introduced by Fisher in the twenties $[5,9]$.

Mandelbrot [2] is one of the first authors that linked statistical physics with the theory of statistical inference, adopting the viewpoint that one can work in statistical mechanics directly with probability distributions over macroscopic variables, the microscopic substructure (e.g., phase space) being largely superfluous. Let $U$ denote the internal energy. Mandelbrot [2] established which is the form of the probability distribution $p_{\gamma}(U)$ that allows for an adequate description of the energy fluctuations of a system in contact with a heat bath at the (inverse) temperature $\gamma=1 / T$. The ensuing distribution turns out to be the celebrated, text-book (Gibbs') canonical one [10], an exponential probability density. A quite interesting uncertainty relation between mean energy and inverse temperature is then obtained (see below). A main protagonist in his treatment is Fisher's information measure $[5,9,11,12]$.

Now, power-law distributions are ubiquitous in physics, critical phenomena being a conspicuous example [13]. In a statistical mechanics' context they arise quite naturally if the 
information measure one maximizes (subject to appropriate constraints) in order to arrive at the equilibrium distribution is not Shannon's one but a generalized one. A lot of work in this respect has been devoted to Tsallis' measure (see [14-19] and references therein).

In view of the importance of these results it should seem appropriate to revisit the FisherMandelbrot link by taking a closer look at non-exponential distributions of the power-law kind. The ensuing results will offer novel insights into the meaning of non-extensivity: Fisher's measure involves all energy moments. Some new features of the thermal uncertainty subject will also be revealed. We proceed first to a brief reminder of Fisher-related concepts.

\section{A BRIEF FISHER PRIMER}

Estimation theory [20] provides one with a powerful result that needs to be quoted before embarking into the present discussion. Consider a system that is specified by a physical parameter $\theta$. Let $\mathbf{x}$ be a stochastic variable and $p_{\theta}(\mathbf{x})$ the probability density for this variable, which depends on the parameter $\theta$. An observer makes a measurement of $\mathbf{x}$ and has to best infer $\theta$ from this measurement, calling the resulting estimate $\tilde{\theta}=\tilde{\theta}(\mathbf{x})$. One wonders how well $\theta$ can be determined. Estimation theory asserts [20] that the best possible estimator $\tilde{\theta}(\mathbf{x})$, after a very large number of $\mathbf{x}$-samples is examined, suffers a mean-square error $e^{2}$ from $\theta$ that obeys a relationship involving Fisher's $I$, namely, $I e^{2}=1$, where the Fisher information measure $I$ is of the form

$$
I=\int d \mathbf{x} p_{\theta}(\mathbf{x})\left\{\frac{\frac{\partial p_{\theta}}{\partial \theta}}{p_{\theta}(\mathbf{x})}\right\}^{2}=\left\langle\left[\frac{1}{p_{\theta}(\mathbf{x})} \frac{\partial p_{\theta}}{\partial \theta}\right]^{2}\right\rangle .
$$

This "best" estimator is called the efficient estimator. Any other estimator must have a larger mean-square error. The only proviso to the above result is that all estimators be unbiased, i.e., satisfy $\langle\tilde{\theta}(\mathbf{x})\rangle=\theta$.

Thus, Fisher's information measure has a lower bound, in the sense that, no matter what parameter of the system we choose to measure, $I$ has to be larger or equal than the inverse of the mean-square error associated with the concomitant experiment. This result, i.e., 


$$
I e^{2} \geq 1
$$

is referred to as the Cramer-Rao (CR) bound, and constitutes a very powerful statistical result [5]. Applications of Fisher's information measure to different physical problems have proliferated in the last 12 years (see details and references in Frieden's book [5]).

\section{THE THERMAL UNCERTAINTY RELATION}

Mandelbrot [2] has established which is the form of the probability distribution $p_{\gamma}(U)$ that allows for an adequate description of the energy fluctuations of a system in contact with a heat bath at the (inverse) temperature $\gamma=1 / T$. It is required that estimators for $\gamma$ should be functions of the energy $U$ only [4] (one demands sufficiency of the estimator [4]). We are led to the canonical distribution

$$
p_{\gamma}(U)=g(U) \frac{e^{-\gamma U}}{Z(\gamma)}
$$

with $Z(\gamma)=\int d U g(U) e^{-\gamma U}$ the partition function and $g(U)$ the structure function, which would be interpreted as a measure of the number of microscopic states compatible with energy $U[4]$.

Let us address the question of estimating the unknown parameter $\gamma$ of the system by measurements of the energy. In this case the Fisher information reads [4]

$$
I(\gamma)=\left(\Delta_{\gamma} U\right)^{2}=\left\langle U^{2}\right\rangle_{\gamma}-\langle U\rangle_{\gamma}^{2}
$$

The CR inequality for unbiased estimators $\tilde{\gamma}$ then yields

$$
\Delta_{\gamma} U \Delta \tilde{\gamma} \geq 1
$$

which is Mandelbrot's uncertainty relation between energy and temperature, expressing that the efficiency with which temperature can be estimated is bounded by the spread in energy. This does not entail that the temperature does really fluctuate. It is assumed throughout that the distribution function (3) with fixed $\gamma$ provides one with an adequate description. Instead, 
the estimators are fluctuating, random quantities. Their standard deviation is employed as a criterion to indicate the quality with which the inverse temperature is estimated [4].

We can translate the preceding considerations into a microscopic, statistical mechanics' language as follows: i) you start with a system in contact with a heath bath at the temperature $T$, described by Gibbs' canonical distribution (3), ii) role switch: regard the associated inverse temperature (originally a variational Lagrange multiplier in the entropy maximization process $[21,22])$ as an estimator, iii) consider the Fisher information for (3) together with its associated CR bound and then, iv) you get a thermal uncertainty relation from this CR bound.

\section{MOTIVATION FOR REVISITING THE THERMAL UNCERTAINTY DERIVATION}

The point we wish to make here is that the above referred to heath bath, employed in Mandelbrot's derivation, cannot be a finite one. It is shown in [17] that if one attempts to repeat Gibbs' celebrated derivation [10] for the probability distribution (PD) that maximizes entropy for a system in contact with a finite heath bath at the inverse temperature $\beta$, the ensuing PD is not Gibbs canonical distribution for the internal energy $U$. Instead, one is forced to deal with the power-law distribution [17]

$$
p(U)=\frac{1}{Z_{q}}[1-\beta(1-q) U]^{\frac{1}{1-q}} ; q \in \Re,
$$

where $Z_{q}$ is a normalization constant (the partition function). For $q=1$ the above $q$ probability distribution becomes Gibbs' canonical one. The distribution (6) maximizes the so-called Tsallis information measure $S_{q}$, whose main feature is that of being non-extensive if $q \neq 1$ : for two independent systems $A, B$ the entropy composition rule is $[14,15]$

$$
S_{q}(A+B)=S_{q}(A)+S_{q}(B)+(1-q) S_{q}(A) S_{q}(B) .
$$

Remember that one of the fundamental tenets of information theory is that of assigning an information content (Shannon's measure) to any normalized probability distribution. 
The whole of statistical mechanics can be elegantly re-formulated by extremization of this measure, subject to the constraints imposed by the a priori information one may possess concerning the system of interest $[21,22]$. It has been shown in the last decade (see, for instance [14-16,18,19] and references therein) that a parallel process can be undertaken with reference to Tsallis' measure, giving rise to what is called non-extensive Tsallis' thermostatistics, responsible for the successful description of an ample variety of phenomena that cannot be explained by appeal to the conventional, extensive one (that of Boltzmann-Gibbs) $[14,15]$.

It is shown in [17] that a system in contact with a finite bath is properly described by a distribution of the type (6). The canonical distribution obtains only in the limit in which the heath bath becomes infinite [17]. In order to repeat the steps described in closing the preceding Section when the protagonist is a power-law PD, one needs to evaluate the associated Fisher measure. What happens then with the associated, putative thermal uncertainty? Will it remain operative? We show below that it will NOT. This entails that the thermal uncertainty cannot be a fundamental physical property. Our task is not a trivial one, as the content of the following section will show.

\section{FISHER MEASURE FOR A POWER-LAW DISTRIBUTION}

We discuss here two different information measures of the Tsallis type, and their associated probability distributions, in order to repeat the steps outlined previously (last paragraph of Section III) that led to a thermal uncertainty relation for exponential distributions. We deal first with the original Tsallis measure and discuss afterwards the concept of escort distribution.

\section{A. Original Tsallis measure}

We start with

$$
p_{\beta}(U(\mathbf{x})) \equiv p_{\beta}(\mathbf{x})=Z_{q}^{-1}[1-(1-q) \beta U(\mathbf{x})]^{\frac{1}{1-q}},
$$


where $\beta$ is a variational Lagrange multiplier and $Z_{q}$ is the accompanying partition function (as we sum over microstates no structure constant is needed [23])

$$
Z_{q}=\int d \mathbf{x}[1-(1-q) \beta U(\mathbf{x})]^{\frac{1}{1-q}}
$$

We effect now the just mentioned role-switch with regards to the meaning of the parameter $\beta$ by introduction of the probability distribution (8) into $I: \beta$ plays the same role as $\gamma$ above. It becomes an estimator:

$$
I=\int d \mathbf{x} p_{\beta}(\mathbf{x})^{-1}\left[\frac{\partial p_{\beta}(\mathbf{x})}{\partial \beta}\right]^{2},
$$

According to Tsallis' tenets [14] i) (Tsallis' cut-off)

$$
(1-q) \beta U(\mathbf{x}) \leq 1
$$

guaranteeing non-negative probabilities and ii) one computes mean-values according to

$$
\langle U\rangle_{q}=\int d \mathbf{x} p_{\beta}^{q} U(\mathbf{x})
$$

For the sake of an easier notation we shall omit, herefrom, writing down explicitly the variable $\mathbf{x}$. We need to evaluate the integrand of Eq. (10). By using (8) one finds that

$$
\frac{\partial p_{\beta}}{\partial \beta}=-p_{\beta}^{q} U Z_{q}^{q-1}-p_{\beta} Z_{q}^{-1} \frac{\partial Z_{q}}{\partial \beta}
$$

and by (9)

$$
\frac{\partial Z_{q}}{\partial \beta}=-Z_{q}^{q}\langle U\rangle_{q}
$$

As a consequence, one has, for the integrand in (10)

$$
p_{\beta}^{-1}\left(\frac{\partial p_{\beta}}{\partial \beta}\right)^{2}=Z_{q}^{2(q-1)}\left[p_{\beta}^{2 q-1} U^{2}+p_{\beta}\langle U\rangle_{q}^{2}-2 p_{\beta}^{q} U\langle U\rangle_{q}\right]
$$

so that, when the above relation is replaced into (10), we arrive at

$$
I=Z_{q}^{2(q-1)}\left[\left\langle p_{\beta}^{q-1} U^{2}\right\rangle_{q}-\langle U\rangle_{q}^{2}\right]
$$

By suitably manipulating Eq. (8), it is now easy to see that 


$$
p_{\beta}^{q-1} Z_{q}^{q-1}=[1-(1-q) \beta U]^{-1}
$$

which allows one to write, for the product of the first two factors in the first term on the right hand side above,

$$
Z_{q}^{2(q-1)} p_{\beta}^{2 q-1}=p_{\beta}[1-(1-q) \beta U]^{-2}
$$

Limiting ourselves to $q$-values such that $|q|<1$, one can expand the last expression into a power series in $(1-q) \beta$ (the convergence of the series is assured because of Tsallis' cut-off):

$$
Z_{q}^{2(q-1)} p_{\beta}^{2 q-1}=p_{\beta} \sum_{n=0}^{\infty}(n+1)(1-q)^{n} \beta^{n} U^{n} .
$$

Inserting this into the first term of the r.h.s of Eq. (15) one finally gets

$$
I=\sum_{n=0}^{\infty}(n+1)(1-q)^{n} \beta^{n} \nu_{n+2}-Z_{q}^{2(q-1)}\langle U\rangle_{q}^{2},
$$

where $\nu_{n+2}=\left\langle U^{n+2}\right\rangle_{q=1}$, is the $n+2-t h$ order momentum of the probability distribution $[20]$.

It is time to give now to the last term of Eq. (19) the form of a momentum expansion. We note first that

$$
\langle U\rangle_{q}^{2} Z_{q}^{2(q-1)}=\left[\left\langle Z_{q}^{q-1} U\right\rangle_{q}\right]^{2}
$$

which, because of (16) can be cast as

$$
\left\langle Z_{q}^{q-1} U\right\rangle_{q}=\left\langle U[1-(1-q) \beta U]^{-1}\right\rangle_{q=1}
$$

Thus, a power-series expansion in $(1-q) \beta$ plus Cauchy's series' multiplication rule yield

$$
\langle U\rangle_{q}^{2} Z_{q}^{2(q-1)}=\sum_{n=0}^{\infty}(1-q)^{n} \beta^{n} \sum_{k=0}^{n} \nu_{k+1} \nu_{n-k+1},
$$

and, finally

$$
I=\sum_{n=0}^{\infty}(1-q)^{n} \beta^{n}\left[\nu_{n+2}-\sum_{k=0}^{n} \nu_{k+1} \nu_{n-k+1}\right]
$$


that involves cross-correlation terms. We would like to establish now an à la Mandelbrot uncertainty (5). This turns out to be impossible! We cannot define a generalized uncertainty that assimilates the second order $U$-momentum to $I$, because the quantity $I$ contains a sum of terms in powers of the estimator $\beta$. This negative result implies that thermal uncertainties are not operative for finite baths, only for (unphysical) infinite ones.

\section{B. A Tsallis-like measure: the "escort" one}

One may wonder whether the peculiar aspect of the mean values $\langle U\rangle_{q}=\int d \mathbf{x} p_{\beta}^{q} U(\mathbf{x})$ may not be responsible for the failure we have just detected. We will repeat now the above steps using ordinary, linear mean values. At this point we introduce the useful concept of escort probabilities (see [24] and references therein). One introduces the transformation

$$
p_{\beta}^{q}(\mathbf{x}) \rightarrow P_{\beta}(\mathbf{x})
$$

with

$$
P_{\beta}(\mathbf{x})=\frac{p_{\beta}^{q}(\mathbf{x})}{\int d \mathbf{x} p_{\beta}^{q}(\mathbf{x})},
$$

$q$ being any real parameter. Here, of course, $p_{\beta}(\mathbf{x})$ is given by (8). For $q=1$ we have $P_{\beta} \equiv p_{\beta}$ and, obviously, $P_{\beta}$ is normalized to unity. Our main theme here is that any fundamental physical law must be invariant under the above transformation.

General global quantities formed with escort distributions of different order $q$, such as the different types of information or mean values, will give more revealing information than those formed with the original distribution only. Changing $q$ is indeed a tool for scanning the structure of the original distribution [24]. However, basic relationships among expectation values, like, say, Ehrenfest theorem, are invariant under the escort transformation.

\section{Heisenberg's uncertainty relations are invariant under (23)}

We start with usual coordinate-momentum relation 


$$
\Delta \widehat{x} \Delta \widehat{p} \geq \frac{\hbar}{2}
$$

where

$$
(\Delta \widehat{x})^{2}=\left\langle\widehat{x}^{2}\right\rangle-\langle\widehat{x}\rangle^{2}
$$

while a similar expression for the momentum fluctuation $\Delta \widehat{p}$.

Expectation values of operators general $\widehat{A}$ are defined as customary

$$
\langle\widehat{A}\rangle=\operatorname{Tr}(\widehat{\rho} \widehat{A})
$$

where $\widehat{\rho}$ is, of course, the density (or statistical) operator.

Under the transformation (23) we have

$$
\widehat{\rho} \rightarrow \frac{\widehat{\rho}^{q}}{\operatorname{Tr}\left(\widehat{\rho}^{q}\right)} \equiv \widehat{\Omega}
$$

so that

$$
\operatorname{Tr}(\widehat{\rho} \widehat{A}) \rightarrow \operatorname{Tr}\left(\frac{\widehat{\rho}^{q} \widehat{A}}{\operatorname{Tr}\left(\widehat{\rho}^{q}\right)}\right) \equiv \operatorname{Tr}(\widehat{\Omega} \widehat{A}),
$$

which entails

$$
\langle\widehat{A}\rangle \rightarrow\langle\widehat{A}\rangle_{e s c} \equiv \operatorname{Tr}(\widehat{\Omega} \widehat{A}),
$$

i.e.,

$$
\Delta \widehat{x}^{(e s c)} \Delta \widehat{p}^{(e s c)} \geq \frac{\hbar}{2}
$$

with

$$
\left(\Delta \widehat{x}^{(e s c)}\right)^{2}=\langle\widehat{x}\rangle_{e s c}^{2}-\left\langle\widehat{x}^{2}\right\rangle_{e s c},
$$

and analogously for $\Delta \widehat{p}^{(e s c)}$.

The form of Heisenberg's principle remains invariant under (23). 
Di Sisto et al. have shown [25] that one can develop an alternative non-extensive thermostatistics that employs an information measure $S_{P P}=S_{P P}\left[P_{\beta}\right]$ which is a functional of the escort distribution of order $q . S_{P P}$ depends upon the escort PD in the same manner as Tsallis' measure depends on the original distribution. The associated mean values are linear in the probabilities. In our case

$$
\langle U\rangle_{e s c}=\int d \mathbf{x} P_{\beta}(\mathbf{x}) U(\mathbf{x}) .
$$

We face now an "escort" Fisher's measure

$$
I=\int d \mathbf{x} P_{\beta}(\mathbf{x})^{-1}\left[\frac{\partial P_{\beta}(\mathbf{x})}{\partial \beta}\right]^{2} .
$$

For our purposes we need to evaluate the integrand in (34). Taking derivatives in (24) we find

$$
\frac{\partial P_{\beta}}{\partial \beta}=q P_{\beta}\left\{p_{\beta}^{-1} \frac{\partial p_{\beta}}{\partial \beta}-\left\langle p_{\beta}^{-1} \frac{\partial p_{\beta}}{\partial \beta}\right\rangle_{e s c}\right\},
$$

so that, taking derivatives in (8) one is led to

$$
\frac{\partial p_{\beta}}{\partial \beta}=-Z_{q}^{q-1} U p_{\beta}^{q}-p_{\beta} Z_{q}^{-1} \frac{\partial Z_{q}}{\partial \beta},
$$

and, using the result

$$
\frac{\partial Z_{q}}{\partial \beta}=-Z_{q}^{q} Q\langle U\rangle_{e s c}
$$

where we have defined $Q=\int d \mathbf{x} p_{\beta}^{q}$, we get

$$
\frac{\partial p_{\beta}}{\partial \beta}=-Z_{q}^{q-1}\left[U p_{\beta}^{q}-p_{\beta} Q\langle U\rangle_{e s c}\right] .
$$

Replacement of this relation into (35) leads to

$$
\frac{\partial P_{\beta}}{\partial \beta}=-q Z_{q}^{q-1} P_{\beta}\left\{p_{\beta}^{q-1} U-\left\langle p_{\beta}^{q-1} U\right\rangle_{e s c}\right\},
$$

and then to 


$$
P_{\beta}^{-1}\left(\frac{\partial P_{\beta}}{\partial \beta}\right)^{2}=q^{2} Z_{q}^{2(q-1)} P_{\beta}\left\{p_{\beta}^{2(q-1)} U^{2}+\left\langle p^{q-1} U\right\rangle_{e s c}^{2}-2 p_{\beta}^{q-1} U\left\langle p_{\beta}^{q-1} U\right\rangle_{e s c}\right\} .
$$

Finally, replacing this into (34), Fisher's information measure acquires the appearance

$$
I=q^{2} Z_{q}^{2(q-1)}\left\{\left\langle p_{\beta}^{2(q-1)} U^{2}\right\rangle_{e s c}-\left\langle p_{\beta}^{q-1} U\right\rangle_{e s c}^{2}\right\}
$$

Recourse to (17) seems to yield now a $2 n d$ order moment. However, it is not a $U$-moment but one of the "effective" energy

$$
E=U /[1-(1-q) \beta U]
$$

i.e.,

$$
q^{-2} I=\mu_{E} \equiv\left\langle E^{2}\right\rangle_{e s c}-\langle E\rangle_{e s c}^{2}
$$

so that the Cramer-Rao bound gives

$$
\mu_{E} \Delta_{\beta} \geq q^{-2}
$$

Appearances are deceptive, though. The above is not an uncertainty relation, because $\beta$ enters the two factors in the l.h.s. Indeed, expansion into a $(1-q) \beta$-powers series and Cauchy's rule, where $\nu_{k}=\left\langle U^{\nu}\right\rangle_{e s c}$ is now a generalized momentum of order $\nu$, with $\nu_{k, k-n}=$ $\nu_{k} \nu_{n-k}$, gives

$$
I=q^{2} \sum_{n=0}^{\infty}(1-q)^{n-1} \beta^{n-1}\left[(n+1) \nu_{n}-\sum_{k=0}^{n} \nu_{k, k-n}\right]
$$

which shows again that it is not possible to get a thermal uncertainty relation between $U$ and $\beta$.

\section{CONCLUSIONS}

As the main result of this Communication we find that it is impossible to find a thermal uncertainty of the type (5) if the underlying probability distribution is not of the exponential form. While for such exponential PDs $I$ can be assimilated to the second moment of the 
energy, for non-exponential PDs (for instance, of the Tsallis form) the Fisher information measure becomes a sum over all energy moments that involves all powers of the estimator $\beta$ as well. This prevents us from re-obtaining the thermal uncertainty relation of Mandelbrot's for non-exponential PDs.

A physical interpretation of the above circumstance is connected with the type of heath bath that helps our system to attain thermal equilibrium. The thermal uncertainty relation only holds for systems in contact with an infinite bath, since only in such a case the Gibbs canonical distribution strictly applies. For finite baths one needs a Tsallis-canonical distribution, as shown in detailed fashion in [17].

Clearly, the status of the thermal uncertainty relation is thereby affected. It can not be regarded as a fundamental property. These facts could constitute a hopefully interesting footnote to the ongoing controversy concerning uncertainty relations in thermodynamics.

\section{ACKNOWLEDGEMENT}

F. Pennini thanks financial support from UNLP. 


\section{REFERENCES}

[1] L. Rosenfeld, in Ergodic theories, edited by P. Caldirola (Academic Press, NY, 1961).

[2] B. Mandelbrot, Ann. Math. Stat. 33, 1021 (1962); IRE Trans. Inform. Theory IT-2, 190 (1956); J. Math. Phys. 5, 164 (1964).

[3] B. Lavenda, Int. J. Theor. Phys. 26, 1069 (1987); 27, 451 (1988); J. Phys. Chem. Sol. 49, 685 (1988); Statistical physics: a probabilistic approach (J. Wiley, NY, 1991).

[4] J. Uffink and J. van Lith, Foundations of Physics 29, 655 (1999).

[5] B. R. Frieden, Physics from Fisher information (Cambridge University Press, Cambridge, England, 1998).

[6] N. Bohr, Collected works, edited by J. Kalckar (North-Holland, Amsterdam, 1985), Vol. 6, pp. 316-330 and 376-377; A. Pais, Niels Bohr's times in physics, philosophy, and polity (Clarendon Press, Oxford, 1991).

[7] C. Kittel, Phys. Today (May 1988) 93.

[8] B. B. Mandelbrot, Phys. Today (January 1989) 71.

[9] B. R. Frieden and B. H. Soffer, Phys. Rev. E 52, 2274 (1995).

[10] J. W. Gibbs, Elementary principles in statistical mechanics (Yale University Press, 1903).

[11] A. R. Plastino and A. Plastino, Phys. Rev. E 54, 4423 (1996).

[12] A. Plastino, A. R. Plastino, and H. G. Miller, Phys. Lett. A 235, 129 (1997).

[13] N. Goldenfeld, Lectures on phase transitions and the renormalization group (AddisonWesley, NY, 1992).

[14] C. Tsallis, Braz. J. of Phys. 29, 1 (1999), and references therein.

[15] A. Plastino and A. R. Plastino, Braz. J. Phys. 29, 50 (1999). 
[16] C. Tsallis, J. Stat. Phys. 52, 479 (1988).

[17] A. R. Plastino, A. Plastino, Phys. Lett. A 193, 251 (1994).

[18] E. M. F. Curado and C. Tsallis, J. Phys. A 24, L69 (1991); Corrigenda: 24, 3187 (1991) and 25, 1019 (1992).

[19] A. R. Plastino and A. Plastino, Phys. Lett. A 177, 177 (1993).

[20] H. Cramer, Mathematical methods of statistics, (Princeton University Press, Princeton, NJ, 1946).

[21] E. T. Jaynes, Phys. Rev. 106, 620 (1957); 108, 171 (1957).

[22] E. T. Jaynes in Statistical Physics, ed. W. K. Ford (Benjamin, New York, 1963);

A. Katz, Statistical Mechanics, (Freeman, San Francisco, 1967).

[23] F. Reif, Statistical and thermal physics (McGraw-Hill, NY, 1965).

[24] C. Beck and F. Schlögl, Thermodynamics of chaotic systems (Cambridge University Press, Cambridge, England, 1993).

[25] R. P. Di Sisto, S. Martinez, R. B. Orellana, A. R. Plastino, and A. Plastino, Physica A 265, 590 (1999). 